\title{
Reforma al Artículo Innumerado Décimo del Código de la Niñez y Adolescencia
}

\section{Reform to the Innumerable Article Ten of the Code of the Childhood and Adolescence}

Jacqueline Cabanilla León ${ }^{a}$

\author{
INFORMACIÓN DEL \\ ARTÍCULO \\ Fecha de recepción: 10 de febrero del \\ 2017 \\ Fecha de aceptación: 15 de Junio del \\ 2017
}

\footnotetext{
${ }^{a}$ Licenciada en Ciencias Políticas y Sociales, Magíster en Derecho Constitucional. Docente de la Facultad de Derecho y Gobernabilidad, Universidad ECOTEC - Ecuador E-mail: jcabanilla@ecotec.edu.ec
}

\begin{abstract}
Resumen
En el Título V artículo inn.10, del Código Orgánico de la Niñez y Adolescencia, existe una errónea aplicación por parte de los jueces, en cuanto al derecho que tiene toda mujer embarazada a reclamar una pensión alimenticia contra el presunto padre, pues, en caso de demostrarse de que no ha sido el padre biológico, no existe ninguna sanción por el perjuicio causado. La autora de este trabajo sugiere que se debe reformar el artículo mencionado, aumentando un inciso que diga que en caso de que al nacer el niño se demostrare que el presunto padre no es el padre biológico y ha estado pagando una pensión alimenticia impuesta por el Juez, el afectado tendrá derecho a reclamar los valores aportados durante el periodo de embarazo a la madre del menor.
\end{abstract}

\section{Palabras Clave:}

Padre presunto, mujer embarazada, pensión alimenticia, niñez y adolescencia, padre biológico.

Clasificación JEL: K360

\begin{abstract}
In title $\mathrm{V}$ article inn.10 of the Organic Code of Children and Adolescents, there is an erroneous application by the judges, regarding the right of every pregnant woman to claim a maintenance pension against the alleged father, If it proves that it has not been the biological father, there is no sanction for the damage caused. The author of this work suggests that the article should be reformed, adding an incision that says that in case the child is shown at the time that the alleged father is not the biological father and has been paying alimony imposed by the judge, The affected person will have the right to claim the values contributed during the period of pregnancy to the mother of the minor.
\end{abstract}

\section{Keywords}

Alleged father, pregnant woman, alimony, childhood and adolescence, biological father.

JEL Classification: K360 


\section{Introducción}

En los actuales momentos en el Ecuador la mujer que demuestre que se encuentra embarazada, puede demandar ayuda pre-natal para exigir el pago de una pensión alimenticia al presunto progenitor.

El artículo 148 del Código Orgánico de la Niñez y Adolescencia tipifica que la mujer embarazada tiene derecho a alimentos, desde el momento de la concepción, para la atención de sus necesidades básicas, y el artículo 149 del mismo Código establece que el presunto padre también está obligado a dar alimentos, por lo cual, el juez competente debe fijar una pensión alimenticia, quedando el presunto padre obligado a pagarla durante todo el tiempo del embarazo de la mujer y ayudando también con un valor adicional para los gastos en el parto.

Según las providencias que a diario se emiten en las unidades judiciales, para fijar una pensión alimenticia a una mujer embarazada solo se está tomando en consideración el hecho de que la mujer indique quien es el posible progenitor de la criatura que lleva en su vientre y demuestre su estado de gestación con el respectivo examen médico.

\section{Marco Teórico}

Ante la existencia de una falta de precisión y omisión de supuestos facticos establecidos en el artículo inn.10 del título V del Código Orgánico de la Niñez y Adolescencia, relacionado con la pensión de alimentos para mujer embara- zada, se encuentra la existencia que de una errónea aplicación del derecho por parte de los jueces, al establecer una pensión alimenticia contra el presunto progenitor, sin estar seguros que él es el padre de la criatura, pues no es dable dejar en indefensión al demandado, vulnerando de esta forma sus derechos a la defensa y causándole un perjuicio económico.

Por tal razón, se ha realizado un estudio profundo sobre el tema, indagando alternativas para que el presunto progenitor en caso de ser demandado por una mujer embarazada, que en el juicio el juez fija una pensión alimenticia que debe pagar durante todo el periodo de gestación, y al nacer la criatura se hace la prueba de ADN la misma que da como resultado que no es su hijo, al menos tenga la opción a un reclamo por vía judicial y se le permita el reintegro de los valores aportados para que no se sienta afectado económicamente.

\section{Pensión Alimenticia para Mujer Embarazada.}

Los alimentos para la mujer embarazada según la ley, es un derecho muy especial, y de ellos depende la vida, salud, nutrición, bienestar de la madre y del niño desde la concepción, asegurando un buen desarrollo del embarazo, el parto y periodo de lactancia.

Las características de los alimentos enfocados a mujer embarazada son: reciprocidad, personal e intransferible, imprescriptibles, sucesivas, inalienables e irrenunciables, divisibles, alternativas. 
Es intransferible porque un niño no puede decir que no quiere ejercer su derecho de alimentos para que se lo den a otro indigente. Es imprescriptible porque no tiene un tiempo definido ni para nacer ni para extinguirse. Las cuotas son fijadas por el juez para ser pagadas en forma sucesivas. Este derecho también es inalienable e irrenunciable, no se puede renunciar bajo ningún concepto. Es divisible porque se puede dividir en varios alimentantes cuando se necesita obligados subsidiarios. Es alternativo puede hacerse en especie o en dinero (Cader \& Viera, 2006).

Este derecho de Alimentos abarca una protección integral para la madre y para el menor que comprende el embarazo, puerperio, lactancia y comienza desde el momento de la concepción. Basta probar el hecho de la concepción para que la madre pueda reclamar el reconocimiento integral del derecho consagrado en el artículo 148 del Código de la Niñez y Adolescencia. Además, en la Constitución de la República del Ecuador, se encuentra reconocida y garantizada la vida, el cuidado y protección de los niños, niñas y adolescentes, desde la concepción, con lo cual existe la obligación de los padres de mantener a un hijo menor de edad desde que se encuentra en el vientre de la madre.

Guillermo Cabanelas conceptualiza a la maternidad futura, real del embarazo y concede a la mujer una protección juridica especial (Cabanellas, 1997).

Por esta razón, y basados en el principio de la vulnerabilidad, el Estado ga- rantiza la protección de las mujeres que deben afrontar solas la llegada de un hijo, pues según estudios realizados por médicos, se ha llegado a la conclusión de que la maternidad enfrenta condiciones de especial fragilidad, con mayores niveles de estrés y ansiedad de la madre antes y durante el embarazo y, como consecuencia, mayor incidencia de prematuridad, bajo peso al nacer, retraso del desarrollo infantil, déficit atencional e hiperactividad, problemas del lenguaje, deficiente competencia social y el comportamiento del individuo para toda la vida (Arcos, Muñoz, \& Sanchez, 2011).

"La Declaración Universal de Derechos Humanos señala que la responsabilidad no es solo de la madre sino también del padre, frente a la llegada de un hijo, ya sea deseado por ambos o no" (Aguilar, 2014). El fundamento legal es la "Convención sobre los derechos del Niño". En esta convención quedó claramente establecido el concepto de "interés superior del niño", cuyo principio ha sido acogido en la Constitución de la República del Ecuador.

Existe la paternidad irresponsable, en la que al quedar embarazada una mujer, el padre no quiere reconocerlo como su hijo y las madres tienen que recurrir a la justicia para reclamar vía judicial el derecho a la paternidad. La prueba que se debe tener para el reconocimiento del hijo es la prueba de ADN, para lo cual se debe esperar el nacimiento de la criatura. En esas circunstancias, la ayuda pre-natal se hace necesaria porque es un apoyo moral, económico y espiritual a la mujer emba- 
razada, previo al nacimiento del hijo y es importante para asegurar un embarazo saludable y se inicia cuando la mujer descubre su estado de gravidez.

El derecho a alimentos lo tiene toda mujer embarazada y se encuentra garantizado dentro de la Carta Magna, Código Civil y Código de la Niñez y Adolescencia. El fundamento es que si la mujer no recibe ayuda en el momento de su embarazo, este puede afectar la salud del niño que está se encuentrra en su vientre, tal como lo indica el Código de la niñez y la adolescencia, en su Art 148:

La mujer embarazada tiene derecho, desde el momento de la concepción, a los alimentos para la atención de sus necesidades de alimentación, salud, vestuario, vivienda, atención del parto, puerperio, y durante el periodo de lactancia por un tiempo de doce meses contados desde el nacimiento del hijo o hija; si la criatura muere en el vientre materno, o el niño o la niña fallece luego del parto, la protección a la madre subsistirá hasta por un período no mayor a doce meses contados desde que se produjo la muerte fetal del niño o niña (Asamblea Nacional, 2009).

La ley busca proteger a la mujer embarazada, puesto que se encuentra en una situación de vulnerabilidad y está dentro del grupo de atención prioritaria según la Constitución de la República. La demanda la puede hacer durante cualquier momento de su embarazo porque se está protegiendo al niño que está por nacer. El fundamento de la demanda está basado en que el presunto progenitor también debe garantizar el cuidado de la mujer durante el embarazo aunque no esté seguro de que es el padre del menor, lo cual lo puede corroborar cuando nazca la criatura y se realice la prueba de $\mathrm{ADN}$.

\section{Existencia de un presunto progenitor.}

Cuando la mujer embarazada no es casada, la ley extiende la obligación de ayuda pre-natal para el presunto progenitor, es decir, al que puede ser el padre pero no existe mayor seguridad, pues no está casado con la madre, ha mantenido con ella relaciones maritales, pero no quiere aceptar la paternidad.

Un embarazo no es sólo responsabilidad de la mujer sino también del presunto progenitor de la criatura, en caso de que la madre se encuentre soltera. Por esta razón, la ley establece que se puede pedir una pensión alimenticia al presunto padre. Para pedir alimentos para mujer embarazada al supuesto padre, se debe demostrar el estado de gestación, con la prueba de embarazo y la mujer debe indicar a quien ella considera como presunto padre.

El artículo 149 del Código de la Niñez y la Adolescencia dice lo siguiente:

Obligados a la prestación de alimentos.- Están obligados a la prestación de alimentos el padre del niño o niña, el presunto padre en el caso del artículo 131 (actual inn.10) y las demás personas indicadas en el artículo 129 (actual inn.5). Si la paternidad del demandado no se encuentra legalmen- 
establecida, el juez podrá decretar el pago de alimentos, provisional y definitivo, desde que en el proceso obren pruebas que aporten indicios precisos, suficientes y concordantes para llegar a una convicción sobre la paternidad o maternidad del demandado. Una vez producido el nacimiento, las partes podrán, solicitar la práctica de las pruebas biológicas a que se refiere el art. 131, con las consecuencias señaladas en el mismo artículo (Asamblea Nacional, 2009).

La ley especifica que el presunto padre debe pagar pensión alimenticia a la mujer embarazada por un año y también dar una ayuda para el parto y lactancia. Una vez que el niño nace, si la duda del presunto progenitor continúa, puede solicitar al juez la prueba de ADN en donde se podría demostrar que él no es el padre biológico.

En vista de que el problema que existe con el tema de presunción de paternidad está en la dificultad de establecer quién es el padre del niño y para eso se hace necesaria la prueba de ADN y existiendo un presunto progenitor, el juez debe ordenar el pago de la pensión alimenticia por todo el tiempo del embarazo, como también fijar un valor para cubrir los gastos del parto.

En el caso de mujer embarazada, el presunto padre no podrá alegar la impugnación del hijo de una mujer embarazada, si él hubiere tenido una relación íntima con ella, aunque haya sido por una sola vez, si va de acuerdo al tiempo del embarazo actual y a la presunción del tiempo de concepción. No cabe alegación por parte del presunto padre, al no tener una prueba convincente lógica y razonablemente para negarlo no podrá evadir esa responsabilidad de padre en las obligaciones de manutención de la mujer embarazada (Sanguil, 2015).

Si del resultado de esta prueba se desprende que el padre que está pagando una pensión alimenticia no es el padre biológico y quiere que se le devuelvan los valores pagados, podrá hacerlo contra el verdadero padre si este aparece. Pero el problema persiste si no aparece el verdadero padre porque el Código de la Niñez y Adolescencia no tipifica sobre cómo se debe proceder en estos casos.

Si se logra demostrar que la demanda fue planteada con dolo, para obtener un tipo de ingreso económico en perjuicio del demandado, se debería establecer la facultad de reembolsar lo indebidamente pagado, pues si no existe vinculo de filiación tampoco existe la obligación, y esto es algo que con la actual normativa no se puede reclamar (Cevallos, 2013).

Por esta razón, se hace necesario revisar el artículo inn. 10 del Código de la niñez y adolescencia y luego hacer una propuesta sobre este tema.

\section{Revisión}

Art. inn.10.- Obligación del presunto progenitor.- El juez fijará la pensión de alimentos a favor del niño, niña o adolescente a una persona cuya filia- 
ción o parentesco en el caso de los demás parientes consanguíneos no ha sido legalmente establecida, de acuerdo con las reglas siguientes:

En el evento de existir negativa por parte del demandado a someterse a las pruebas de ADN que el juez disponga, se presumirá de hecho la filiación o relación de parentesco en el caso de los demás parientes consanguíneos, con el alimentario y en la misma providencia se fijará la pensión provisional, la cual será exigible desde la presentación de la demanda. b) (...) Se prohíbe practicar los exámenes de ADN al que está por nacer

Si el resultado del examen de ADN es positivo, el Juez declarará la filiación y la correspondiente paternidad o maternidad y dispondrá la inscripción de la respectiva Resolución en que así lo declare el Registro Civil (...)

Si el demandado o demandada funda su negativa para la práctica del examen de $\mathrm{ADN}$ en la circunstancia de carecer de recursos para sufragarlo, el Juez dispondrá que el Ministerio de Salud Pública, a través de la Unidad de Investigación Genética, realice el examen de $\mathrm{ADN}$ en forma gratuita.

Se admitirá la demostración de la carencia de recursos del presunto padre, madre o pariente consanguíneo obligado a sufragar los gastos que demande el examen de ADN, así como las costas procesales y los y los gastos del estudio social, cuando del estudio de la oficina técnica se proba- re dicho particular y de conformidad con la prueba que se actúe en la audiencia respectiva.

En este artículo se demuestra que cuando el demandado tiene dudas de ser el padre del hijo ya nacido, puede solicitar la prueba de ADN, pero no hace mención a qué sucedería en el caso de alimentos para la mujer embarazada, ni indica cómo debe procederse en el caso de que el presunto padre no sea el padre biológico del niño, existiendo de esta forma un vacío legal, pues no se especifica ninguna sanción si esto sucediera.

El artículo inn. 3 del mismo Código de la niñez y adolescencia estipula que el derecho de alimentos no admite reembolso de lo pagado, lo cual impide al demandado solicitar la devolución del pago indebido de pensiones alimenticias.

En el código vigente no se ha establecido la facultad de solicitar la devolución de las pensiones pagadas indebidamente, cuando se demuestre que no hay vínculo parento-filial, lo cual constituye inseguridad jurídica, pues en estos casos si no hay una causa lícita en la obligación, no debería existir el vínculo de filiación ni la obligación de prestar alimentos (Ceva1los, 2013).

El Código de la Niñez y Adolescencia en su artículo inn.5 inciso 4 estipula que los parientes que hubieren realizado el pago podrán ejercer la acción de repetición de lo pagado contra el padre y/o la madre, pero no dice nada sobre el presunto progenitor sino que solamente menciona a los obligados subsidiarios, 
que son los familiares que a falta de padre o madre se hacen cargo de la obligación, pudiendo ser los abuelos, hermanos o tíos, según el Código Orgánico de la niñez y adolescencia.

El Código Civil establece una acción de reembolso y de sanción con la indemnización de daños y perjuicios, pero al estar en inferior jerarquía que el Código Orgánico de la Niñez y Adolescencia, no se puede plantear dicha acción, que tiene fundamento valido en el principio de seguridad jurídica $\mathrm{y}$ en el fundamento de las obligaciones alimenticias que es la filiación o parentesco (Cevallos, 2013).

Con todos estos antecedentes, y ante la existencia de un vacío legal, la autora de este artículo ha realizado una propuesta de Reforma al Código Orgánico de la Niñez y Adolescencia, la cual protege los derechos del presunto progenitor.

\section{Propuesta de Reforma al Artículo inn.10 del Código de la Niñez y Adoles- cencia}

La alternativa que se propone en este trabajo es aumentar un literal más al Art. inn.10 del Código Orgánico de la Niñez y Adolescencia, que especifique cómo se procedería en caso de que habiendo un presunto progenitor pagado alimentos a una mujer embarazada y habiendo ayudado económicamente para el parto, al realizarse la prueba de ADN se demostrare que él no es el padre biológico del niño.
La reforma propuesta por la autora de este artículo quedaría de la siguiente manera en el código de la niñez y adolescencia:

Art. Inn.10.- (...) d) En caso de que en la prueba del examen de ADN se estableciera que el presunto padre no es el padre biológico del niño, y habiendo éste cumplido con la ayuda pre-natal durante todo el tiempo del embarazo de la madre al igual que dando una ayuda para pagar el parto, y no apareciendo el padre biológico del menor, podrá el padre presunto solicitar una devolución a la madre de los valores aportados durante todo ese tiempo. En caso de que el padre biológico apareciera y reconozca al menor, el presunto padre podrá pedir al padre biológico la devolución de lo pagado por concepto de ayuda pre-natal y pensiones alimenticias si fuera el caso.

Con esta propuesta se pretende implementar una responsabilidad a la madre en caso de que dolosamente hubiera perjudicado a una persona que no es el verdadero padre de la criatura que está por nacer. Esto es posible debido a que podría existir confusión de fechas o simplemente mala intención, por lo cual es necesario que la madre asuma su responsabilidad también y que al pedir alimentos para mujer embarazada esté completamente segura de quien es el padre de la criatura.

Reflexionando sobre el tema se podría pensar que se puede ejercer acción de repetición de lo pagado contra el padre en 
en los casos de que un obligado subsidiario cumpla con la obligación de los alimentos, por lo cual, también se podría ejercer la misma acción de repetición contra el verdadero padre en el caso de que el presunto progenitor hubiera pagado alimentos a una mujer embarazada, siendo el problema actual que esto no se encuentra tipificado en la normativa vigente. Por esta razón se hace necesario proponer una reforma al artículo inn. 10 del código de la niñez y adolescencia.

Con esta reforma planteada lo que se pretende es que el presunto progenitor no vea vulnerado su derecho a la defensa y pueda tener la oportunidad de reclamar vía judicial el reembolso correspondiente del dinero pagado en caso de que se demuestre que no sea el verdadero padre del menor y hubiere pagado pensión alimenticia por todo el tiempo del embarazo de la madre y a su vez ayudado en el parto.

\section{Conclusiones}

La autora de este artículo, después de un profundo análisis del tema expuesto, llega a la conclusión de que existe un vacío legal en el Art. inn.10 del Código Orgánico de la Niñez y Adolescencia por tres razones importantes:

1.-No se encuentra establecido en el artículo inn.10, qué sucedería en el caso de la mujer embarazada, una vez que la criatura ha nacido y se ha realizado la prueba de ADN, el resultado hubiere sido que el presunto progenitor no es el verdadero padre de la criatura.
2.- Falta la existencia de un "literal" adicional que exactamente especifique cómo debe procederse en el caso expuesto en el inciso anterior y se propone aumentar un "literal d)" al artículo inn.10 del mencionado Código, en donde se estipule claramente las sanciones que debería tener la madre del niño, en caso de que al realizarse la prueba de ADN resulte que el presunto padre no es el padre biológico de la criatura.

3.- Con este nuevo literal se propone reformar el vacío existente en el Código Orgánico de la Niñez y Adolescencia, de tal forma que si fuere el caso, el padre presunto tiene derecho a reclamar, contra la madre, los valores aportados durante todo el periodo de embarazo y el juez deberá concederle la acción de repetición, que es el derecho para el reclamo de un pago indebido.

\section{Referencias}

Aguilar, L. (2014). Universidad Nacional de Loja. Obtenido de dspace: http://dspace.unl.edu.ec/jspui/bitstream/123456789/6012/1/Luis\%20D iego\%20Aguilar\%20Quizhpe.pdf

Arcos, E., Muñoz, L., \& Sanchez, X. (2011). Revista médica de Chile. Obtenido de Scielo: http://www.scielo.cl/scielo.php?pi$\mathrm{d}=$ S0034-98872011000600007\&script $=$ sci_arttext

Asamblea Nacional. (2009). Código de la Niñez y Adolescencia. Quito: Catedra. 
Asamblea Nacional. (2008). Constitución de la República del Ecuador. En Asamblea. Quito: Catedra.

Asamblea Nacional. (2009). Código de la Niñez y Adolescencia. En Asamblea. Quito: Catedra.

Cabanellas, G. (1997). Diccionario Juríco de Derecho usual. Buenos Aires: Heliasta.

Cader, R., \& Viera, R. (2006). Universidad Francisco Gavidia. Obtenido de ufg.edu.sv: http://ri.ufg.edu.sv/j $\mathrm{s} \quad \mathrm{p}$ u $\mathrm{i} / \mathrm{b}$ i $\mathrm{t}$ s tream/11592/6643/1/346.016\%20 6-C122d.pdf

Cevallos, J. (2013). dspace. Obtenido de http://dspace.unl.edu.ec/jspui/bitstream/123456789/6166/1/Jos\%C3\%A9\%20Francisco\%20R am $\% \mathrm{C} 3 \% \mathrm{~B} 3 \mathrm{n} \% 20 \mathrm{Cevallos} . \mathrm{pdf}$

Clarin Argentina. (20 de septiembre de 2012). Clarin.com. Obtenido de Clarin: http://www.clarin.com/sociedad/Codigo-civil-protecc i o n - e m b a r a zadas_0_778122272.html

Dema, V. (20 de 09 de 2012). La Nación. Obtenido de www.lanacion.com.ar: http://www.lanacion.com.ar/1509878-madres-so1 a s - e 1 - p a d r e - e s ta-obligado-a-garantizar-alimentos -a-la-mujer-embarazada
Kuffo, S., \& Olaya, L. (2015). Repositorio Universidad Laica Vicente Rocafuerte. Obtenido de Universidad Laica Vicente Rocafuerte: h t t p : / / r e pos i torio . u 1 vr.edu.ec/handle/44000/593

Larrea, J. (2008). Los Alimentos Legales. En J. L. Holguin, Manual elemental del Código Civil Ecuatoriano. Quito: Catedra.

Ojeda, R. (08 de junio de 2016). El Universal Colombia. Obtenido de w w w. e 1 u nivers a 1 . c o m : http://www.eluniversal.com.co/consultorio/familia/alimentos-favor-de-joven-embarazada

Olaya, S. K. (2015). Repositorio Universidad Laica Vicente Rocafuerte. Obtenido de www.repositorioulvr.edu.ec: http://repositorio.ul$\mathrm{v} \mathrm{r}$. e d u. e c / b i t s tream/44000/593/1/T-ULVR-0474.pdf

Ortemberg, H. \&. (2012). Código Civil: Protección para mujeres embarazadas.

Ortiz, R. (08 de junio de 2016). El Universal Colombia. Obtenido de eluniversal: http:/www.eluniversal.com.co/consultorio/familia/alimentos-favor-de-joven-embarazada

Palacios, M., \& Coloma, C. (2012). Repositorio Universidad Laica Vicente Rocafuerte. Obtenido de repositorioulvcr: http://repositorio.ulvr.edu.ec/bitstream/44000/511/1/T-ULVR-0448.pdf 
Sanguil, I. (2015). Universidad Nacional de Loja. Obtenido de dspace: http://dspace.unl.edu.ec/jspui/bitstream/123456789/8488/1/Irma\%20Monserrath\%20Sanguil\% 20Villacis.pdf

Unicef. (1946-2006). Convención sobre los derechos del niño. Obtenido de convenciondederechosdelniño: http://www.un.org/es/events/childrenday/pdf/derechos.pdf

Universo Jus diccionario. (01 de agosto de 2015). Universo Jus. Obtenido de universojus.com: http://universojus.com/codigo-civil-comercial-comentado/articulo-665

Zambrano, S. (27 de diciembre de 2008). Abogados Ecuador. Obtenido de abogadosecuador.com: https://abogadosecuador.wordpress.c o m / $2008 / 12 / 27 / \mathrm{impu}$ tar-la-paternidad/ 